\title{
Coinfección pulmonar por Aspergillus fumigatus y Pneumocystis jirovecii en un paciente con VIH-SIDA.
}

(Lung coinfection by Aspergillus fumigatus and Pneumocystis jirovecii in an HIV-AIDS patient)

Debbin Brogca S ${ }^{1}$. María José Valenzuela $\mathbf{P}^{2}$. Gonzalo Wilson $\mathrm{L}^{3}$. Rodrigo Cruz $\mathrm{Ch}^{4}$.

${ }^{1}$ Universidad de Valparaíso, Becada Medicina Interna.

2 Universidad de Valparaíso, Becada Medicina Interna.

${ }^{3}$ Hospital Carlos van Buren de Valparaíso, Infectología

${ }^{4}$ Hospital Carlos van Buren, Infectología.

Universidad de Valparaíso, Cátedra de Micología.

*Autor para correspondencia: debbinb@yahoo.es

Recibido:17-12-12

Aprobado:26-12-12

Palabras claves: Aspergillus fumigatus, Pneumocystis jirovecii, coinfeccion.

Keys words:Aspergillus fumigatus, Pneumocystis jirovecii, coinfection.

\section{RESUMEN}

Se presenta un caso de coinfección pulmonar por Aspergillus fumigatus y Pneumocystis jirovecii en un paciente con VIH-SIDA. Se diagnosticó con

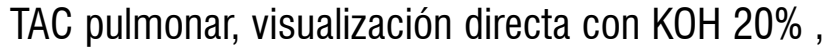
tincion de Gomori-Grocott y cultivo del LBA, galactomanano en sangre y de LBA. Se discuten los factores de riesgo, diagnóstico y tratamiento para cada infección.

\section{INTRODUCCIÓN}

La aspergilosis es una infección oportunista provocada principalmente por Aspergillus fumigatus, sin embargo otras especies como A. flavus, $A$. nidulans o $A$. níger se aíslan cada vez con mayor frecuencia $(1,2)$. Se considera que en pacientes VIHSIDA es una enfermedad subdiagnosticada, problema puesto de manifiesto en un estudio autópsico en que se demostró aspergilosis invasora en un $5,09 \%$ del total de pacientes(3). Los datos analizados en el estudio «Adult and Adolescent Spectrum of HIV Disease Project» mostraron 228 casos de aspergilosis entre 35.252

\section{ABSTRACT}

We report a case of lung coinfection by Aspergillus fumigatus and Pneumocystis jirovecii in a patient with HIV-AIDS. Was diagnosed with lung TAC, direct visualization with $\mathrm{KOH} 20 \%$, GomoriGrocott staining and culture of BAL, galactomannan in blood and BAL. We discuss risk factors, diagnosis and treatment for each infection.

personas con VIH/SIDA (3). La localización más habitual es la pulmonar y se presenta clínicamente como una neumonía necrotisante subaguda 0 crónica (4). Esta infección ocurre en el contexto de un paciente $\mathrm{VIH} / \mathrm{SIDA}$ con recuento de linfocitos T$\mathrm{CD} 4<50$ céls/ml,con otras infecciones oportunistas y sin terapia antirretroviral, o en aquellos con neutropenia o con uso de corticoterapia prolongada $(1,5)$.

La neumonía por Pneumocystis jirovecii es una de las infecciones oportunistas más frecuentes en el paciente VIH y es considerada marcadora de SIDA $(5,6)$. Ocurre principalmente en individuos con recuentos de linfocitos T-CD4 
Coinfección pulmonar por Aspergillus fumigatus y Pneumocystis jirovecii en un paciente con VIH-SIDA - D. Brogca et al.

menores de $200 \mathrm{cels} / \mathrm{ml}$ y es considerada un factor de riesgo para infección por Aspergillus $\operatorname{spp}(7)$. Entre el 13 y $18 \%$ de los pacientes con neumonía por $P$. jirovecii pueden cursar con una coinfección, ya sea por bacterias como Streptococcus pneumoniae, micobacterias u hongos filamentosos como Aspergillus spp (5,8,9).

\section{CASO CLÍNICO}

Paciente de sexo masculino, de 51 años de edad, con antecedentes mórbidos de tabaquismo crónico detenido hace 1 año,TBC pulmonar tratada en 1990, neumonía bacteriana en el 2011 y VIH(+) confirmado en abril del 2012. En Mayo del 2012 realizó su primer control en el policlínico del Hospital Carlos van Buren, destacando una carga viral de 310.000 copias $(\log 6,49)$ y $\mathrm{CD} 4$ de $1 \mathrm{cel} / \mathrm{ml}$. El 5 de junio se hospitaliza por cuadro de 3 semanas de evolución, caracterizado por compromiso del estado general, fiebre, tos con expectoración, dolor epigástrico y candidiasis oral. Entre los exámenes de laboratorio destacó una proteína $\mathrm{C}$ reactiva de $31,7 \mathrm{mg} / \mathrm{dl}$, LDH de $276 \mathrm{U} / \mathrm{L}$ y una B- 2microglobulina de $3 \mathrm{mg} / \mathrm{Lt}$.Se planteó como hipótesis diagnóstica un síndrome febril, con observación de linfoma 0 tuberculosis y VIH/SIDA etapa C3. Se inició tratamiento profiláctico con cotrimoxazol forte 1 comprimido día vía oral y se solicitó TAC tórax,abdomen y pelvis, endoscopía digestiva alta y baciloscopías en expectoración. El 6 de junio se confirmó candidiasis esofágica, por lo que se inició tratamiento con fluconazol $150 \mathrm{mg}$ día vía oral. El 8 de junio sufre un quiebre en su evolución, con cuadro de disnea de rápida instalación, polipnea y mayor compromiso del estado general, por lo que se planteó probable neumonía por Pneumocystis jirovecii o tromboembolismo pulmonar, iniciandose tratamiento con ceftriaxona 1 gr iv/día, cotrimoxazol simple 3 ampollas iv/8 hrs y Dalteparina sódica 5000 U subcutánea/12 hrs. Se le realizó angio TAC de tórax con el cual se descartó un tromboembolismo pulmonar, pero se observaron imágenes en vidrio esmerilado en lóbulos superiores, sugerentes de neumonía por Pneumocistis jirovecii (Imagen 1 der ) y una condensación con cavitacion a nivel retrocardiaco izquierdo (Imagen 1 izq). Se suspende anticoagulación y se mantiene tratamiento con ceftriaxona y cotrimoxazol iv. El día 11 de junio el paciente se encontró en buenas condiciones generales, con menor disnea y polipnea, hemodinamia estable, pero febril, por lo que se decide realizar fibrobroncoscopía (FBC) con lavado broncoalveolar para estudio microbiológico. En la Tinción de Gomori-Grocott se observó gran cantidad de hifas septadas en ángulo dicotómico (Imagen 2 izq), moderada cantidad de Pneumocystis jirovecii (Imagen 2 der) y en el cultivo, en menos de 24 hrs abundante desarrollo de Aspergillus fumigatus (Imagen 3 izq y 3 der). El test de galactomanano en sangre tuvo un indice de 1.3 y el de lavado broncoalveolar de 2.4, por lo que se agregó al tratamiento Anfotericina B iv, con buena respuesta clínica. A los 20 días de tratamiento con Anfotericina se cambió a Itraconazol vo $400 \mathrm{mg}$ día y se inició tratamiento antirretroviral (TARV) con Tenofovir/ emtricitavina y Raltegravir, el cual fue bien tolerado. Con 33 días de tratamiento antifúngico y 12 días de TARV se decide darlo de alta con control en policlínico de infectologia del Hospital Carlos van Buren. 
Coinfección pulmonar por Aspergillus fumigatus y Pneumocystis jirovecii en un paciente con VIH-SIDA - D.Brogca et al

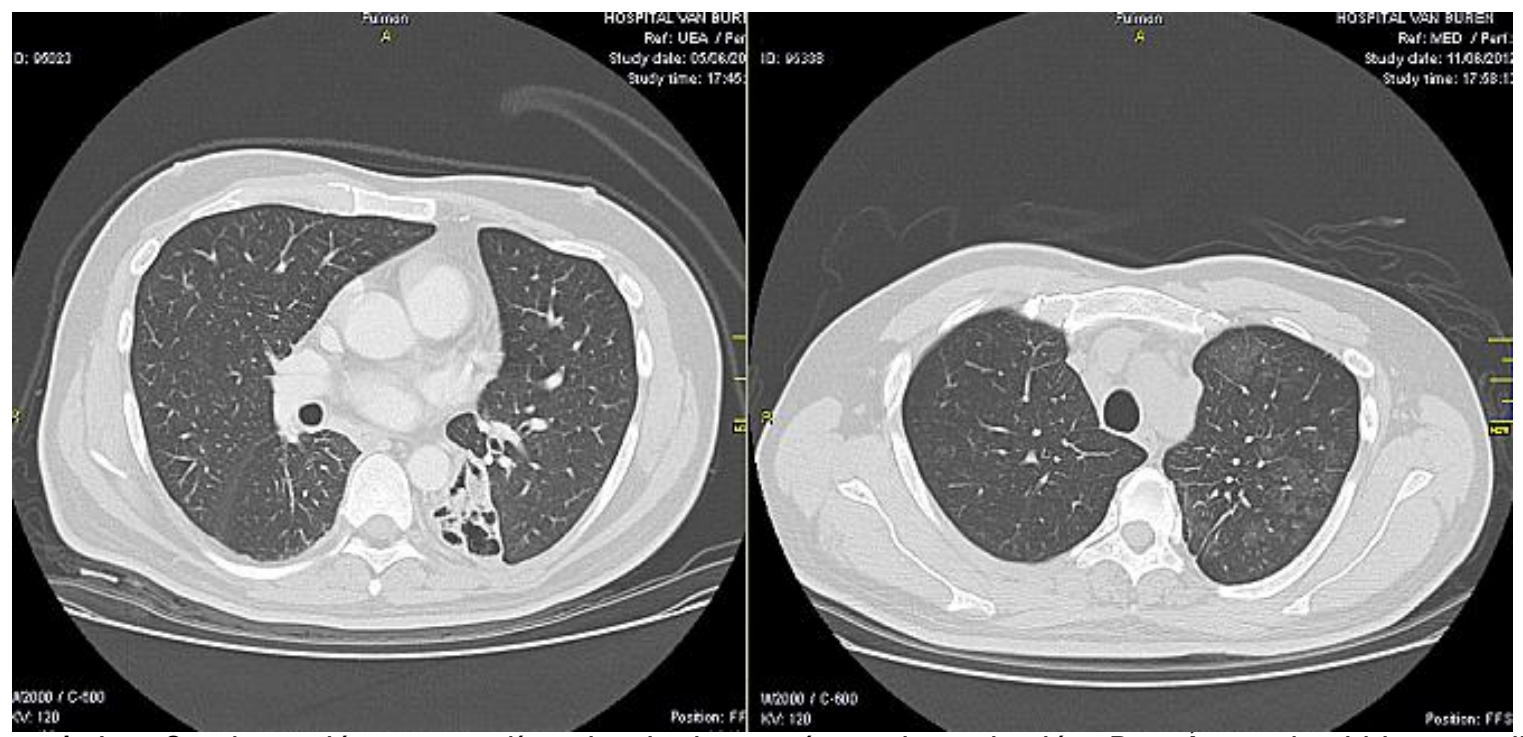

Figura 1. Izq: Condensación retrocardíaca izquierda con áreas de cavitación. Der: Areas de vidrio esmerilado en los lóbulos superiores de ambos campos pulmonares

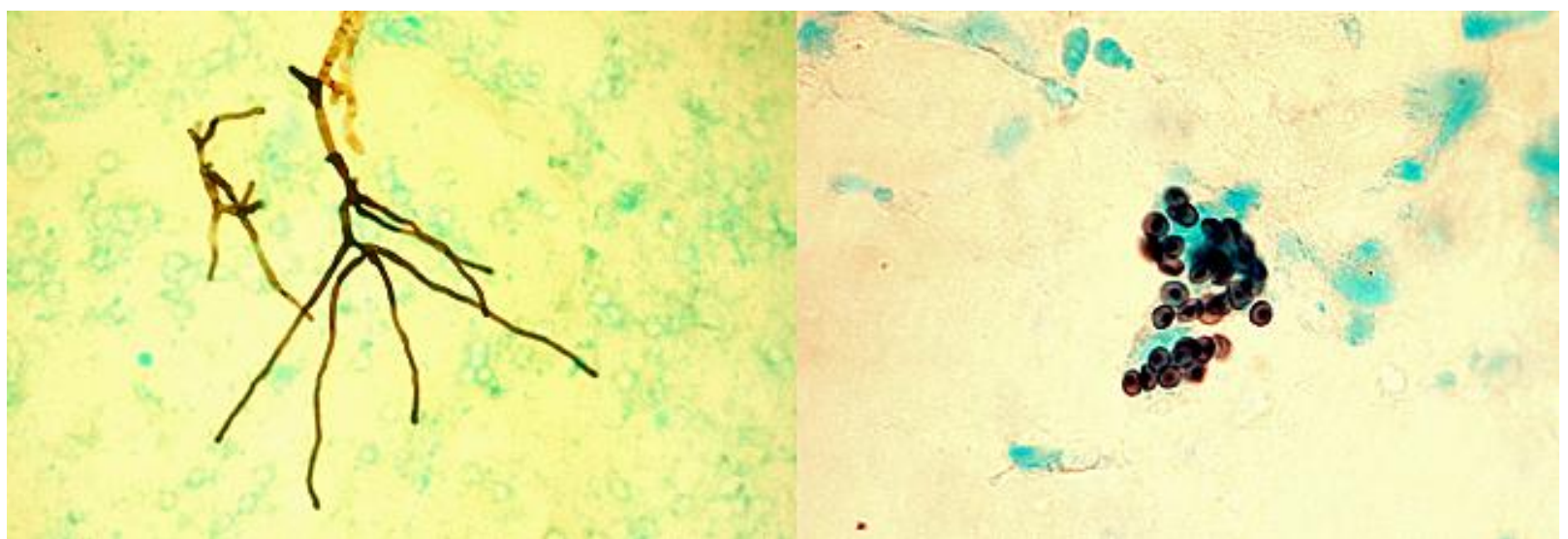

Figura 2. Izq: Se observan hifas septadas en ángulo dicotomico. Tinción de Gomori-Grocott. 40X. Der: Cluster de Pneumocystis jirovecii. Tinción de Gomori-Grocott. 40X.
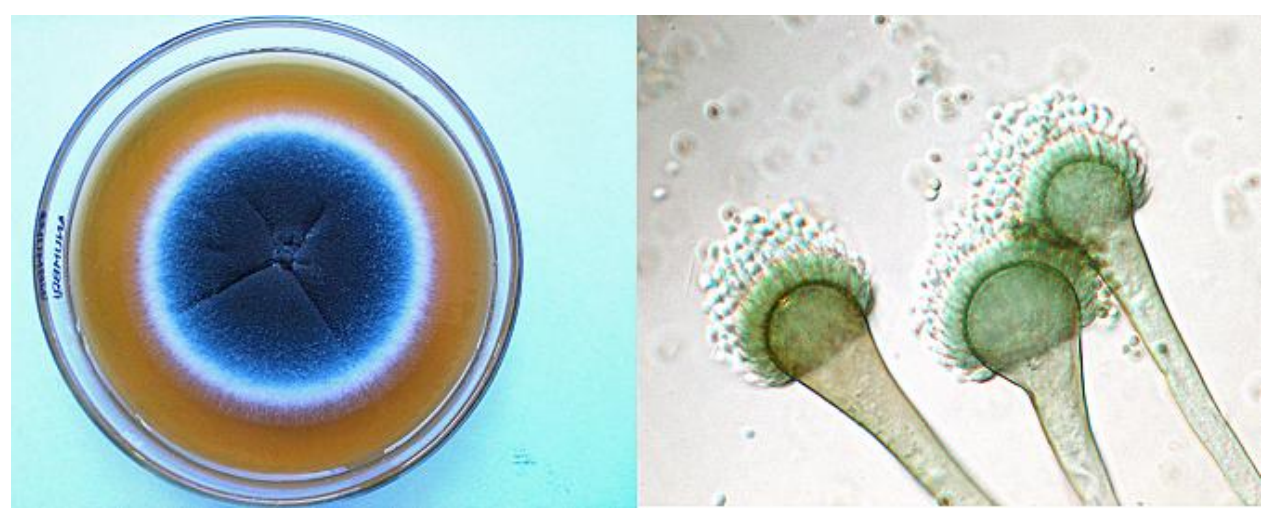

Figura 3. Izq: Cultivo de Aspergillus fumigatus en agar PDA a $37^{\circ} \mathrm{C}$. Der: Vesículas monoseriadas estrictas de Aspergillus fumigatus. Tinción de lactofenol con azul de algodón. $100 \mathrm{X}$. 
Coinfección pulmonar por Aspergillus fumigatus y Pneumocystis jirovecii en un paciente con VIH-SIDA - D. Brogca et al.

\section{DISCUSIÓN}

La coinfección de Aspergillus fumigatus y Pneumocystis jirovecii se ha documentado bien en pacientes no VIH-SIDA con dosis altas de corticoides y uso de metotrexate $(10,11,12)$.

En pacientes con VIH-SIDA también puede ocurrir esta coinfección, por lo que se recomienda la búsqueda de ambos agentes en forma dirigida, especialmente en aquellos pacientes con recuento de linfocitos T-CD $4<50 \mathrm{cel} / \mathrm{ml}$ y sin TARV, o en aquellos con neutropenia 0 que reciben medicamentos inmunosupresores $(5,7,9)$. La relativa lentitud de la infección por Aspergillus en el contexto de la enfermedad VIH es probablemente debido a que la función fagocítica de las células que acompañan a la disfunción de las células T está relativamente conservada(13). El diagnóstico se establece por el cuadro clínico más imágenes de TAC pulmonar y la visión directa de hifas septadas dicotómicas en ángulo de $45^{\circ}$ con cultivos positivos para Aspergillus spp $(14,15)$.

El galactomanano es un polisacárico componente de la pared celular que es liberado por las distintas especies de aspergillus durante la invasión del micelio. El test de ELISA «en sandwich» para galactomano es ampliamente usado como parte del diagnóstico de aspergilosis en pacientes neutropénicos post quimioterapia y en trasplantados $(16,17)$. En pacientes VIH-SIDA podría ser de utilidad, sin embargo aún faltan estudios para aclarar esto (18).

En pacientes oncohematologicos el voriconazol ha sido considerado la terapia de elección, ya que ha demostrado tener menor mortalidad y menos efectos adversos, sin embargo en pacientes $\mathrm{VIH}$ se debe considerar las posibles interacciones de este medicamentos con la TARV, en especial con los inhibidores de proteasas, por lo que la Anfotericina $B$ es una buena alternativa en estos pacientes $(19,20)$.

Pneumocystis jirovecii es uno de los patógenos oportunistas más importantes que afectan a individuos con síndrome de inmunodeficiencia adquirida en etapa SIDA y pacientes inmunocomprometidos por otras causas $(5,6,21)$.
El método clásico de diagnóstico es por medio de la expectoración inducida o el lavado bronco-alveolar. La Tincion de Gomori-Grocott presenta una buena sensibilidad y especificidad, además de ser barata y relativamente fácil de implementar en los laboratorios (22). Otras tinciones como Giemsa 0 azul de toluidina también pueden ser útiles, pero su sensibilidad es menor(22). La inmunofluorescencia directa es una técnica sensible y no dependiente del operador como las tinciones $(22,23)$. Las técnicas de biología molecular resultan promisorias, sin embargo, hay que recordar que un porcentaje de la población está colonizada con este microrganismo, por lo que los resultados deben ser interpretados con cautela, considerando además el cuadro clínico y las imágenes del TAC pulmonar(24,25).

El fármaco de elección para el tratamiento de la neumonía por $P$. jirovecii es la combinación de trimetoprim y sulfametoxazol (cotrimoxazol). En los casos graves con falla respiratoria $(\mathrm{PaO} 2<70$ $\mathrm{mmHg} 0$ una A-a mayor de 35) es perentorio el uso de corticosteroides (25).

La aspergilosis pulmonar invasora y la neumonía por Pneumocystis jirovecii son enfermedades oportunistas que ponen en peligro la vida de los pacientes VIH-SIDA. La sospecha clínica,el diagnóstico dirigido y precoz, con un tratamiento temprano son esenciales para la sobrevida de estos pacientes.

\section{REFERENCIAS}

1. Cruz R, Piontelli E. Enfermedad fúngica invasora (EFI) en pacientes de cinco hospitales de la quinta región de Valparaíso, Chile. 2004 a 2009. Rev Chil Infect 2011; 28 (2): 123-9.

2. Patterson T F, Kirkpatrick W R, White M,
Hiemenz J W, Wingard J R, Dupont B, et al. Invasive aspergillosis. Disease spectrum, treatment practices, and outcomes. Medicine (Baltimore) 2000; 79: 250 60.

\section{Antinori S, Nebuloni M, Magni C, Fasan M, Adorni F, Viola A. Trends in the postmortem diag- nosis of opportunistic invasive fungal infections in}


Coinfección pulmonar por Aspergillus fumigatus y Pneumocystis jirovecii en un paciente con VIH-SIDA - D. Brogca et al.

patients with AIDS: a retrospective study of 1,630 autopsies performed between 1984 and 2002. Am J Clin Pathol 2009; 132 (2): 221-7.

4. Lortholary 0, Meyohas M C, Dupont B, Cadranel J, Salmon-Ceron D, Peyramond D, et al. Invasive aspergillosis in patients with acquired immunodeficiency syndrome: report of 33 cases. Am J Med 1993; 95: 177-87.

5. Lasso M. Diagnóstico y tratamiento de infecciones oportunistas en el paciente adulto con infección por VIH/SIDA. Rev Chil Infect 2011; 28 (5): 440-60.

6. Stansell J D, Osmond D H, Charlebo E, Lavange L, Wallace JM , Alexander BV, et al. Predictors of Pneumocystis carinii pneumonia in HIV-infected persons. Pulmonary complications of HIV infection study group. Am J Respir Crit Care Med 1997; 155: 60-6.

7.Wallace JM, Lim R, Browdy BL, Hopewell PC, Glassroth J, Rosen MJ, et al. Risk factors and outcomes associated with identification of Aspergillus in respiratory specimens from persons with HIV disease. Pulmonary Complications of HIV Infection Study Group. Chest 1998;114:131-7.

8. De los Santos A, Romero P, García JC, Caro N, Girón JA. Micosis en pacientes neutropénicos e inmunosuprimidos. Medicine 2010;10(53):3569-74.

\section{Denning DW, Follansbee SE, Scolaro M, Norris}

S, Edelstein H, Stevens DA. Pulmonary aspergillosis in the acquired immunodeficiency syndrome. N Engl J Med 1991;324:654-62.

10. Mariñosa M, Soler A, Nogués $X$, Pedro-Botet J. Pulmonary coinfection by Pneumocystis carinii and Aspergillus fumigatus in a seronegative arthritis patient treated with low-dose methotrexate. Clinical Rheumatology 2004;23(6) :555-6.

11. Kaira K, Shinomiya Y, Takahashi Y, lida T, Yanagitani N, Sunaga $N$ et al. Coinfection of invasive pulmonary aspergillosis and pneumocystis jirovecii pneumonia in a non-HIV patient.Acta Med Okayama 2007;61(4):235-8.

12. Shankar E.M, Kumarasamy N, Rajan R, Balakrishnan P, Solomon S, Devaleenol B, et al. Aspergillus fumigatus, Pneumocystis jirovecii, Klebsiella pneumoniae \& Mycoplasma fermentans co-infection in a HIV infected patient with respiratory conditions from southern India. Indian J Med Res 2006;123: 181-4.

13. Khoo SH, Denning DW. Invasive aspergillosis in patients with AIDS. Clin Infect Dis. $1994 ; 19$ (1):41-8

14. Cruz R, Vieille P, Fuentes D, Ponce E, Piontelli L.Micosis pulmonares en pacientes de la Quinta Región. Período 2007-2010. Rev Med Chile 2012; 140(5): 595-601.

15. De los Santos A, Romero P, García JC, Caro N , Girón JA. Micosis en pacientes neutropénicos e inmunosuprimidos. Medicine 2010;10(53):3569-74.

16. Maertens J, Verhaegen J, Lagrou K, Van Eldere J, Boogaerts M. Screening for circulating galactomannan as a noninvasive diagnostic tool for invasive aspergillosis in prolongued neutropenic patients and stem cell trasnplantation recipients: a prospective validation. Blood 2001; 97: 1604-10.

18. Maertens J, Verhaegen J, Demuynck H, Brock P, Verhoef G, Vandenberghe P, et al. AutopsyControlled Prospective Evaluation of Serial Screening for Circulating Galactomannan by a Sandwich Enzyme-Linked Immunosorbent Assay for Hematological Patients at Risk for Invasive Aspergillosis. J Clin Microbiol 1999; 37(10): 32238.

18. Leeflang M, Debets-Ossenkopp J, Visser E, Scholten J.P.M, Hooft L, Bijlmer A, et al.Determinaciones de galactomanano para la aspergilosis invasiva en pacientes inmunocomprometidos. The Cochrane Library 2009. 
Coinfección pulmonar por Aspergillus fumigatus y Pneumocystis jirovecii en un paciente con VIH-SIDA - D. Brogca et al.

19. Herbrecht R, Denning D, Patterson T, Bennett

$\mathrm{J}$, Greene R, Oestmann J, et al. For the Invasive Fungal Infections Group of the European Organisation for Research and Treatment of Cancer and the Global Aspergillus Study Group. Voriconazole versus Amphotericin B for Primary Therapy of Invasive Aspergillosis. N Engl J Med 2002; 347:408415.

20. Serrano JI. Interacciones farmacologicas de los nuevos antirretrovirales. Farm Hosp 2011;35(1):3643.

21. Bahamondes L, Villar M, Orellana C. González J. Características de la neumonía por Pneumocystis jirovecii en adultos con SIDA con y sin terapia antiretroviral. Rev Chil Infect 2006; 23 (3): 215-219.

22. Vieille P, Fuentes D. Métodos diagnósticos tintoriales para Pneumocystis jirovecii. Bol Micol 2011; 26 :29- 34.
23.Khan M. A, Farrag N, Butcher P.Diagnosis of Pneumocystis jiroveciii pneumonia: immunofluorescence staining, simple PCR or nPCR.Journal of Infection 1999; 39(1): 77-80.

24. Jarboui M A, Sellami A, Sellami H, Cheikhrouhou F, Makni F, Arab N B, et al. Molecular diagnosis of Pneumocystis jirovecii neumonía in immunocompromised patients. Mycoses 2010;53(4):329-333.

25. Guidelines of the AIDS Study Group (GESIDA)/ National AIDS Plan. Treatment of Opportunistic Infections in Adolescent and Adult Patients Infected by the Human Immunodeficiency Virus During the Era of Highly Active Antiretroviral Therapy. Enferm Infecc Microbiol Clin 2008;26(6):356-79. 